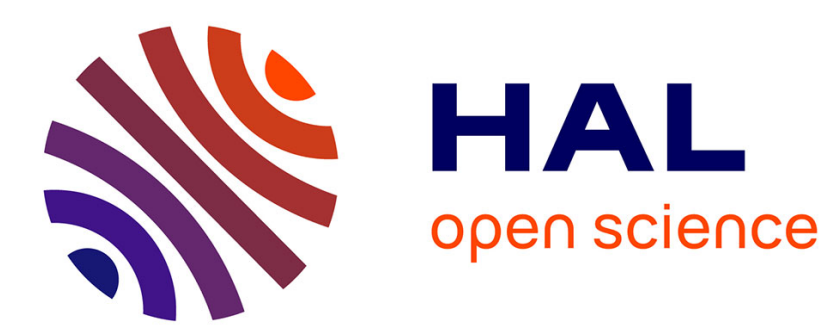

\title{
Self-diffusion and Inter-diffusion Coefficients of Liquid Lithium Isotopes \\ Marco Omini
}

\section{To cite this version:}

Marco Omini. Self-diffusion and Inter-diffusion Coefficients of Liquid Lithium Isotopes. Philosophical Magazine, 2006, 86 (12), pp.1643-1666. 10.1080/14786430500482328 . hal-00513641

\section{HAL Id: hal-00513641 \\ https://hal.science/hal-00513641}

Submitted on 1 Sep 2010

HAL is a multi-disciplinary open access archive for the deposit and dissemination of scientific research documents, whether they are published or not. The documents may come from teaching and research institutions in France or abroad, or from public or private research centers.
L'archive ouverte pluridisciplinaire HAL, est destinée au dépôt et à la diffusion de documents scientifiques de niveau recherche, publiés ou non, émanant des établissements d'enseignement et de recherche français ou étrangers, des laboratoires publics ou privés. 


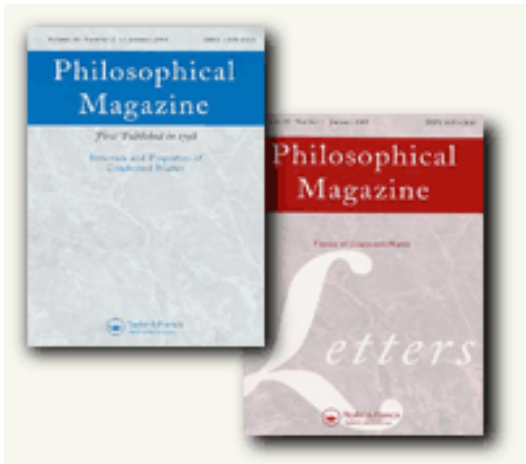

\section{Self-diffusion and Inter-diffusion Coefficients of Liquid Lithium Isotopes}

\begin{tabular}{|r|l|}
\hline Journal: & Philosophical Magazine \& Philosophical Magazine Letters \\
\hline Manuscript ID: & TPHM-05-Jul-0339 \\
\hline Journal Selection: & Philosophical Magazine \\
\hline $\begin{array}{r}\text { Date Submitted by the } \\
\text { Author: }\end{array}$ & 18-Jul-2005 \\
\hline Complete List of Authors: & Omini, Marco; Politecnico of Torino, Physics \\
\hline Keywords: & diffusion, liquid metals \\
\hline Keywords (user supplied): & isotope effect \\
\hline
\end{tabular}

\section{S) ScholaroNE \\ Manuscript Central}




\title{
SELF-DIFFUSION AND INTER-DIFFUSION COEFFICIENTS OF LIQUID LITHIUM ISOTOPES
}

\author{
M. Omini \\ Dipartimento di Fisica del Politecnico di Torino, Italy
}

\begin{abstract}
.
The experimental data on the $\mathrm{Li}^{6}$ and $\mathrm{Li}^{7}$ diffusivities, as given by Feinauer, Majer and Seeger (1994), are discussed in the frame of a theory previously developed by the author (1986). The scattering processes responsible for diffusion are described in terms of a Fourier- transformable pair potential through which the diffusing atom interacts with the atoms of the surrounding liquid. Two main improvements are introduced into the theory with respect to its original formulation: the first is represented by a more reliable form of trial function in the variational procedure used to solve the transport equation, the second consists in a reinterpretation of the long wavelength part of the potential, that is the part connected with collective modes of vibration. The theory provides an explanation of the dynamic isotope effects observed in self- diffusion and inter-diffusion coefficients.
\end{abstract}




\section{1- Introduction.}

In a previous paper [1] the problem of self-diffusion in liquid metals was treated in the frame of a pseudopotential formulation, successively extended to liquid metal alloys [2]. The two above papers, hereafter referred to as I and II, respectively, have been considered with particular attention in recent years, after the experimental work of Feinauer, Majer and Seeger (FMS) [3-4]: in fact these authors determined the ratio between the self-diffusivities of $\mathrm{Li}^{6}$ and $\mathrm{Li}^{7}$ and found a value in full agreement with the prediction of the theory contained in I. The same theory was also quoted as a possible source of explanation of isotope effects observed in more complex liquids [5]. However, a reading of papers I and II leaves some problems open: these concern $a$ ) the reliability of the simple trial function employed there as a solution of the transport equation; $b$ ) the temperature dependence of the diffusivities ; $c$ ) the relation between the diffusivities of $\mathrm{Li}^{6}$ in pure $\mathrm{Li}^{7}$ and of $\mathrm{Li}^{7}$ in pure $\mathrm{Li}^{6}$. For instance, with reference to point $b$ ), FMS point out that the strong thermal rise of the self-diffusivities, as observed in lithium between $454 \mathrm{~K}$ and $580 \mathrm{~K}$, remains unexplained. In a more general way, it has to be concluded that the FMS data related to points $b$ ) and $c$ ) require an interpretation in terms of a new version of the theory. This new version is precisely the aim of the present paper: it will contain on one hand an exhaustive discussion of point $a$ ), on the other hand a clarification of the model potential adopted in I and a careful analysis of its relation with the collective modes of the liquid: the presence of these modes, which was overlooked in the previous works, is the key to understand the temperature behaviour of the self-diffusion coefficients.

\section{2- Summary of the previous work and suggestions for an improved trial function.}

In this section we outline the basic assumptions and results of the theory developed in I. We consider a system of atoms with mass $m_{a}$ (solute atoms) dissolved in a host liquid composed of $N$ atoms with mass $m_{b}$ (solvent atoms). The concentration of solute atoms is so small that their mutual interaction can be neglected. Let $j$ be an index numbering the atoms of the host liquid $(j=1,2, \ldots N)$. The interaction between a solute atom at $\boldsymbol{r}$ and a solvent atom at $\boldsymbol{R}_{j}$ is described in terms of a Fouriertransformable potential $\varphi\left(\boldsymbol{r}-\boldsymbol{R}_{j}\right)$. This is assumed sufficiently soft to allow the analysis of any scattering process in the frame of the Born approximation applied to plane wave states. The starting point is the probability rate for a process in which a solute atom with wavevector $\boldsymbol{q}$ is scattered into a wavevector $\boldsymbol{q}^{\prime}=\boldsymbol{q}+\boldsymbol{K}$, owing to its interaction with a dynamical state of the host liquid characterized by atoms with wavevectors $\boldsymbol{Q}_{1}, \boldsymbol{Q}_{2}, \ldots \boldsymbol{Q}_{N}$. Calling $\Omega$ the volume of the box containing the liquid, we used in I for the above probability rate the expression

$$
\left(2 \pi / \hbar \Omega^{2}\right) \Phi^{2}(K) \Sigma_{j} \delta\left[\varepsilon_{a}(\boldsymbol{q})+\varepsilon_{b}\left(\boldsymbol{Q}_{j}\right)-\varepsilon_{a}(\boldsymbol{q}+\boldsymbol{K})-\varepsilon_{b}\left(\boldsymbol{Q}_{j}+\boldsymbol{K}\right)\right]
$$

where 


$$
\Phi(K)=\int \mathrm{d}^{3} r \exp (-i \boldsymbol{K} \cdot \boldsymbol{r}) \varphi(r)
$$

and $\varepsilon_{s}(\boldsymbol{q})=\hbar^{2} q^{2} / 2 m_{s}$, with $s=a, b$. We present in Appendix A a rigorous justification of this expression, as deducible from the principles of quantum mechanics: in fact in I it was only obtained through qualitative arguments. After averaging over the initial states of the host liquid, we deduced the probability rate of interest for transport theory: this was written in the form $\Omega^{-1} P\left(\boldsymbol{q}, \boldsymbol{q}^{\prime}\right)$, where

$$
P\left(\boldsymbol{q}, \boldsymbol{q}^{\prime}\right)=\left(2 \pi m_{b} / k_{\mathrm{B}} T\right)^{1 / 2} v^{-1} \hbar^{-2} K^{-1} \Phi^{2}(K) \exp \left\{-\lambda_{b} K^{-2}\left[\mu(\boldsymbol{K} \cdot \boldsymbol{q})+(1+\mu) K^{2} / 2\right]^{2}\right\}
$$

and $\mu=m_{b} / m_{a}, \lambda_{s}=\hbar^{2} /\left(2 m_{s} k_{\mathrm{B}} T\right), v=\Omega / N$.

We assume the existence of a fictitious force $\boldsymbol{F}_{a}$ acting on the solute atoms, so that they represent a system in a steady state condition, where the diffusive contribution to the total current balances the drift contribution. Calling $n(\boldsymbol{q}) \mathrm{d}^{3} q$ the number of solute atoms per unit volume with wavevector in $\mathrm{d}^{3} q$ and introducing the rate $S\left(\boldsymbol{q}, \boldsymbol{q}^{\prime}\right)=$ $P\left(\boldsymbol{q}, \boldsymbol{q}^{\prime}\right) \exp \left(-\lambda_{a} q^{2}\right)$, which was shown to be symmetric with respect to the exchange of $\boldsymbol{q}$ and $\boldsymbol{q}^{\prime}$, we take from I the following form of the Boltzmann equation:

$$
-\left(\lambda_{a} / \pi\right)^{3 / 2} \boldsymbol{V}_{\boldsymbol{q}} \cdot\left[\nabla \mathcal{N}_{a}-\left(k_{\mathrm{B}} T\right)^{-1} \mathcal{N}_{a} \boldsymbol{F}_{a}\right] \exp \left(-\lambda_{a} q^{2}\right)=\Omega^{-1} \Sigma_{q^{\prime}} S\left(\boldsymbol{q}, \boldsymbol{q}^{\prime}\right)\left[\psi_{\boldsymbol{q}}-\psi_{q^{\prime}}\right]
$$

where $\boldsymbol{V}_{\boldsymbol{q}}=\hbar \boldsymbol{q} / m_{a}$ and $\psi_{\boldsymbol{q}}$ is related to $n(\boldsymbol{q})$ through the definition

$$
n(\boldsymbol{q})=\left[\left(\lambda_{a} / \pi\right)^{3 / 2} \mathcal{N}_{a}+\psi_{q}\right] \exp \left(-\lambda_{a} q^{2}\right)
$$

$\mathcal{N}_{a}$ being the total number of solute atoms per unit volume.

Eq. (4) shows that the value of $\boldsymbol{S}\left(\boldsymbol{q}, \boldsymbol{q}^{\prime}\right)$, and consequently of $P\left(\boldsymbol{q}, \boldsymbol{q}^{\prime}\right)$, corresponding to $\boldsymbol{q}^{\prime}=\boldsymbol{q}$, is never called into play: actually, in such a case the square bracket on the right hand side vanishes. This justifies the fact that the analysis required to arrive at eq. (3), as developed in Appendix A, can be confined to $\boldsymbol{K} \neq 0$. Putting now

$$
\nabla \mathfrak{N}_{a}-\left(k_{\mathrm{B}} T\right)^{-1} \mathfrak{N}_{a} \boldsymbol{F}_{a}=X \boldsymbol{U}
$$

where $\boldsymbol{U}$ is a unit vector along the concentration gradient, we deduced in I the equation

$$
-\left(\lambda_{a} / \pi\right)^{3 / 2} X \Sigma_{\boldsymbol{q}}\left(\boldsymbol{V}_{\boldsymbol{q}} \cdot \boldsymbol{U}\right) \psi_{\boldsymbol{q}} \exp \left(-\lambda_{a} q^{2}\right)=(2 \Omega)^{-1} \Sigma_{\boldsymbol{q}, \boldsymbol{q}^{\prime}}\left(\psi_{\boldsymbol{q}}-\psi_{\boldsymbol{q}^{\prime}}\right)^{2} S\left(\boldsymbol{q}, \boldsymbol{q}^{\prime}\right)
$$

which is suitable for the use of variational techniques. More precisely, choosing for $\psi_{q}$ a trial function depending on a set of adjustable parameters, one determines these parameters in such a way that the right hand side of eq. (7) is a maximum, under the constraint represented by eq. (7) itself.

Transforming the right hand side of eq. (4) into an integral over $\boldsymbol{K}$ and introducing dimensionless variables $y=\lambda_{a}^{1 / 2} K, u=\lambda_{a}^{1 / 2} q$, it is possible to show (see Appendix $\mathrm{B}$ of I ) that the unknown solution of eq. (4) must be of the form 


$$
\psi_{q}=-C X G\left(\lambda_{a}^{1 / 2} q\right)(\boldsymbol{q} \cdot \boldsymbol{U})
$$

where $C$ is a positive constant and $G(u)$ a function satisfying the integral equation

$$
u=\int_{0}^{\infty} y B(y) \mathrm{d} y \int_{-1}^{1}[u G(u)-(u+y \xi) G(t)] \exp (-\mathcal{K}) \mathrm{d} \xi
$$

in which the following definitions are used :

$$
\begin{gathered}
B(y)=[\Phi(K) / \Phi(0)]^{2}=\left[\Phi\left(\lambda_{a}^{-1 / 2} y\right) / \Phi(0)\right]^{2} \\
t(\xi, y, u)=\left(u^{2}+y^{2}+2 u \xi y\right)^{1 / 2} \\
\mathcal{K}(\xi, y, u)=\mu^{-1}[\mu u \xi+(1+\mu) y / 2]^{2}
\end{gathered}
$$

The current density of solute atoms is

$$
\boldsymbol{J}_{a}=\int \boldsymbol{V}_{\boldsymbol{q}} n(\boldsymbol{q}) \mathrm{d}^{3} q=(2 \pi)^{3} \Omega^{-1} \Sigma_{\boldsymbol{q}} \psi_{\boldsymbol{q}} \boldsymbol{V}_{\boldsymbol{q}} \exp \left(-\lambda_{a} q^{2}\right)
$$

Substituting expression (8) into eq. (13) provides $\boldsymbol{J}_{a}$ as a sum of a diffusion term and a drift term, namely

$$
J_{a}=-D \nabla \mathscr{N}_{a}+\mu \mathfrak{N}_{a} \boldsymbol{F}_{a}
$$

the Einstein relation $\mu=D / k_{\mathrm{B}} T$ being automatically verified.

For consistency, $G(u)$ is expected to be always positive. If, for some value of $q$, such a condition were not satisfied, the particles with velocity $\hbar q / m_{a}$ would give rise to a drift current contribution having opposite direction with respect to the force $\boldsymbol{F}_{a}$ : the friction coefficient presented by the liquid to these particles would be negative!

The calculations performed in I were confined to the choice $G(u)=$ constant. As shown in Appendix B, this can be considered as the solution of eq. (9) in the long wavelength limit $(u \rightarrow 0)$. The high $q$ behaviour of the solution of eq. (9) is examined in Appendix $\mathrm{C}$ : in the limit $u \rightarrow \infty$ the function $G(u)$ is shown to be proportional to $u^{3}$. Therefore, the simplest trial function satisfying the asymptotic behaviours for $u \rightarrow 0$ and $u \rightarrow \infty$ can be written in the form

$$
\psi_{q}=\left(\alpha+\beta u^{3}\right)(\boldsymbol{q} \cdot \boldsymbol{U})=\left(\alpha+\lambda_{a}^{3 / 2} \beta q^{3}\right)(\boldsymbol{q} \cdot \boldsymbol{U})
$$

where $\alpha$ and $\beta$ can be determined through a variational procedure applied to eq. (7).

\section{3- Pseudopotential and collective motion.}

In the present formulation a central role is played by the pair potential $\Phi(K)$. To throw light on this function, let us consider an alternative description of the liquid 
system, in which normal modes of collective motion are introduced. Such a description is equivalent to assuming the existence of phonons in liquids [6-8]. Of course, only a fraction of the $3 N$ degrees of freedom can be accomodated into normal modes, otherwise the liquid would not differ from a crystal lattice: the remaining degrees must be connected with single particle motions. It has to be believed that the above fraction is small, as suggested by the good agreement with experiment of Ziman's formula for electrical resistivity [9]. Such a formula, in fact, does not take into account electronphonon interaction, which in a solid metal is the main source of resistivity: the only possible explanation of this agreement is that in a liquid metal the number of phonon modes is small.

If phonons are present, the vibrational contribution to the free energy is of the form

$$
k_{\mathrm{B}} T \Sigma_{K} \ln \left[1-\exp \left(-\hbar \omega_{K} / k_{\mathrm{B}} T\right)\right]
$$

where the sum is over all the wavevectors $\boldsymbol{K}$ corresponding to the above modes. The total free energy (including, in addition to (16), the contribution of single particle motions) must be minimized with respect to all the variables on which it depends. In particular, for a given number $N^{\prime}$ of vectors $\boldsymbol{K}$ participating to (16), it depends on the choice of these vectors: there are, in fact, infinite ways to construct a sample of $N^{\prime}$ vectors of the reciprocal space. This sample plays the role of a variable intrinsical to the vibrational contribution and, therefore, must be chosen in such a way that this contribution is a minimum. If the frequency $\omega_{K}$ is assumed to increase with $K$, this is also true for each term of the sum (16): as a consequence, the smaller the length of the vectors of the sample, the lower the value taken by the sum. This means that the minimum will be reached by packing the $N^{\prime}$ vectors with the highest possible density inside a sphere surrounding the origin of the reciprocal space. The conclusion is that the collective modes are associated to all the vectors $\boldsymbol{K}$ for which $K<K_{m}$, where $K_{m}$ is the radius of the above sphere. Since $N^{\prime}$ is presumed to be small, $K_{m}$ must necessarily be lower than the Debye radius $Q_{\mathrm{D}}=\left(6 \pi^{2} / v\right)^{1 / 3}$.

It has to be stressed that the above modes, as deduced from minimization of the free energy, refer to a liquid in thermodynamical equilibrium. Modes with any $K$ higher than $K_{m}$ can be excited, however only through experiments which typically bring the system into non-equilibrium conditions (anelastic neutron or $\mathrm{X}$ ray scattering experiments $[10,11])$.

We also note that the degrees of freedom pertaining to normal modes are in competition with those related to single particle motions, which are favoured by an increase of disorder, that is of $T$. For this reason $N^{\prime}$, and therefore $K_{m}$, is expected to decrease when the temperature is raised.

In a description accounting explicitly for phonon modes, let us now think of the expression equivalent to (3), giving the probability rate for a scattering process in which the solute atom exchanges a momentum $\hbar \boldsymbol{K}$ with the surrounding liquid. A substantial contribution to this expression is precisely expected to derive from the interaction between the solute atom and the collective mode with wavevector $\boldsymbol{K}$ (collective interaction). Such an interaction is in competition with a process in which the above momentum is directly exchanged between the solute atom and one of the surrounding neighbours (local interaction). The curve representing the probability rate 
vs $K$ is expected to show a marked drop for $K \sim K_{m}$, owing to the absence of collective modes beyond this value. Such a behaviour must be reflected in $\Phi^{2}(K)$, to which the present theory commits the whole description of the interaction between the diffusing atom and the liquid. Therefore we can write

$$
\Phi^{2}(K)=\chi_{c}(K)+\chi_{l}(K)
$$

where $\chi_{c}(K)$ accounts for the whole effect of collective modes (including a term due to the interference between local interaction and collective interaction, and responsible for a quantum mechanical contribution to the probability rate of scattering): therefore $\chi_{c}(K)$ vanishes in the interval $K>K_{m}$, where only the local interaction term $\chi_{l}(K)$ survives. Since the values of $K$ corresponding to thermal energies are at least one order of magnitude higher than $K_{m}$, it follows that in the range of the $K$ axis of interest for liquid metals $\chi_{c}(K)$ is a short range function, confined to a small interval near the origin.

The decomposition (17) means that diffusion is a complex phenomenon, where both the collective and the local interaction must be considered in order to describe in a correct way the behaviour of the diffusing particle (solute atom). So far only the latter interaction has been explicitly treated in the literature, in other words only the collisions of the particle with one of the neighbours have been assumed to be relevant. However, the failure of the classical models to explain the dynamic isotope effect led Buchhauser et al [5] to suggest the importance of collective phenomena in the theory of diffusion. The term represented by $\chi_{c}(K)$ is precisely the answer of the present formulation to their suggestion.

The physical meaning of $\chi_{c}(K)$ can be better understood through the following picture, which is also useful to clarify the definition of phonon in the liquid state. Since liquids are characterized by a short range order, the collective modes interacting with the diffusing particle must be confined to a region of limited extent around it, involving only a few shells of neighbours. The collisions described by $\chi_{c}(K)$ are precisely scattering events in which the particle, through a resonance process, exchanges energy and momentum with the elementary collective excitations present in such a short range environment, to which approximately all our previous considerations concerning phonons can be referred. As we shall see, these collisions represent the only form of scattering mechanism when $K$ is small, since in this case $\chi_{c}(K)$ is enormously higher than $\chi_{l}(K)$ (see sect. 4).

The technical possibility of accounting for collective motions through the simple presence of a function like $\chi_{c}(K)$ is understandable by the help of Appendix D (point a). To describe the interaction between the diffusing particle and the collective modes it is not necessary to introduce explicitly these modes into the theory. It is sufficient to treat the diffusion process through a liquid where no collective modes exist, but the interaction between the diffusing particle and any atom of the liquid is described by a potential whose Fourier transform is a short range function on the $K$ axis, confined to a small interval near $K=0$ : the result is the same as if we explicitly accounted for the interaction between the particle and a system of phonons with wavevectors lying in the same interval. The proof represents a strong argument in favour of our simple procedure. 
This equation can be considered an important long wavelength limit condition for the pseudopotential of our interest. As shown by the above authors, the collective modes associated to eq. (18) are characterized by isothermal sound velocities: this is precisely the property we expect for the phonons with $K<K_{m}$, which refer to a liquid in thermodynamical equilibrium. Therefore eq. (18) will be preferred to the corresponding form used in I, where the presence of a factor containing the specific heat ratio was suitable to characterize adiabatic velocities.

\section{4 - Pseudopotential and mean free path.}

$\chi_{c}(K)$ does not play any role in the determination of the mean free path $l$ of the diffusing atom, because the values of $K$ for which $\chi_{c}(K)$ is appreciably different from zero refer to scattering processes where the wavevector change, as measured by $K$, is much lower than the wavevector of the atom before the interaction: in fact the latter is of the order of the thermal average $Q_{t h}=\left(3 k_{\mathrm{B}} T m_{a}\right)^{1 / 2} / \hbar$, which for $\mathrm{Li}^{7}$ at the melting point is about $11 Q_{\mathrm{D}} \gg K_{m}$. Such processes are low angle collisions, that help the atom to escape from the cage of its neighbours and travel through the liquid (high mobility processes). On the contrary, the low mobility processes are head-on collisions (large angle scattering) which produce localization maintaining the atom inside the cage. Since the reciprocals of the mean free paths are added when two competing mechanisms are present, the resulting value of $l$ is essentially the mean free path associated with large angle collisions, which correspond to values of $K$ where only the local interaction is active. These arguments are supported by the calculation contained in Appendix D (point $b$ ), where the mean free path due to the 
interaction with the collective modes is estimated to be much higher, presumably by a factor of the order of 50 .

The contribution to $l$ of the local interaction can be deduced by the simple kinetic formula

$$
l=[N \mathcal{S} / \Omega]^{-1}
$$

where $\mathcal{S}$ is the average cross section presented to the diffusing atom by an atom of the host liquid. This formula holds when $l$ is much larger than the range of the interaction between the colliding particles. For this reason it could not be applied to the collective interaction, because $\chi_{c}(K)$ is limited to a small interval of the $K$ axis, so that its inverse Fourier transform is a long range function in the direct space. Of course, the use of the same formula for the local interaction requires to verify that in such a case its conditions of validity are fulfilled: this check is postponed to the end of the present section.

The calculation of $S$ was tackled in I: one starts from the differential scattering cross section presented by the host liquid to a diffusing atom with wavevector $\boldsymbol{q}$, namely $\Omega V_{\boldsymbol{q}}^{-1} \cdot \Omega^{-1} P(\boldsymbol{q}, \boldsymbol{q}+\boldsymbol{K}) \cdot \Omega(2 \pi)^{-3} \mathrm{~d}^{3} K$, and obtains $N \mathcal{S}$ (macroscopic cross section) by integrating over $\boldsymbol{K}$ and averaging over $\boldsymbol{q}$. Let us introduce a function $\varphi_{l}(r)$ defined in such a way that its Fourier transform $\Phi_{l}(K)$ satisfies the condition $\Phi_{l}{ }^{2}(K)=\chi_{l}(K)$ for any $K$. As a consequence, in the case of selfdiffusion $\left(m_{a}=m_{b}=m\right.$ ), employing the usual dimensionless variables $y$ and $u$, we obtain from eq. (3) $s=2 m^{2} I /\left(\pi^{2} \hbar^{4}\right)$, where

$$
I=\int_{0}^{\infty} \Phi_{l}^{2}\left(\lambda_{a}^{-1 / 2} y\right) y \mathrm{~d} y \int_{0}^{\infty} u \mathrm{~d} u \int_{-1}^{1} \exp \left[-u^{2}-(y+u \xi)^{2}\right] \mathrm{d} \xi
$$

It is convenient to express the mass of the diffusing atom in terms of the difference $\Delta=Z_{n}-Z_{e}$ between the number of neutrons and the number of electrons that it contains. Neglecting the small difference between neutron mass and proton mass, that is putting $m_{p}=m_{n}=1.84 \cdot 10^{3} m_{e}$ and expressing the electron mass $m_{e}$ in terms of the Fermi energy $\varepsilon_{F}$, we deduce from eq. (19) the following formula for the mean free path $l$

$$
l^{-1}=\left(1+\Delta / 2 Z_{e}\right)^{2} l_{\mathrm{o}}^{-1}
$$

where

$$
l_{\mathrm{o}}^{-1}=6.28 \cdot 10^{7} v^{-1 / 3} Z_{V}^{4 / 3}\left(Z_{e} / v \varepsilon_{\mathrm{F}}\right)^{2} I
$$

and $Z_{V}$ is the valence of the atoms.

To obtain a reasonable representation of $\Phi_{l}$, let us start from a liquid of heavy atoms, for which $Z_{e} \gg \Delta$ and $l$ is coincident with $l_{0}$. In this case large angle scattering processes are favoured, because the rigid sphere diameters are large (see Table 1): therefore each atom can be thought to perform a random motion inside the 
cage of its neighbours, from which it cannot escape. As a consequence, $l$ is expected to be essentially determined by the free volume at disposal of the atom inside the cage: an approximate formula corresponding to such a picture is $l=\xi R_{A}$, where $R_{A}=$ $(3 v / 4 \pi)^{1 / 3}$ denotes the radius of the atomic volume and $\xi$ lies between 0.1 and 0.2 [13]. The important point is that $l$ is related to a microscopic property which, at constant volume, is temperature- independent: this parallels a classical result of the gas theory, for which $l$ is predicted to be independent of $T$. These simple considerations suggest to choose $\Phi_{l}(K)$ in such a way that the integral (20) appearing in (22) does not contain $T$. This is possible in two cases:

i) $\Phi_{l}\left(\lambda_{a}^{-1 / 2} y\right)$ depends on $y$, but not on $T$;

ii ) $\Phi_{l}\left(\lambda_{a}^{-1 / 2} y\right)$ is a constant with respect to both $y$ and $T$.

Case $i$ ) corresponds to assuming $\Phi_{l}(K)=f\left(T^{-1 / 2} K\right)$. Although in principle an explicit temperature dependence cannot be excluded for a pseudopotential, however such an assumption is ruled out by its inconsistency in the limit $K \rightarrow \infty$. In fact in this limit $\Phi_{l}(K)$ must be coincident with the Fourier transform of the real potential, which of course cannot be temperature dependent. The only reasonable choice is therefore represented by $i$ ) : in this case $\Phi_{l}(K)$ is a constant, say $A$, independent of $K$ and $T$. The independence of $K$ implies, in particular, that $A$ must also represent the asymptotic behaviour of $\Phi_{l}(K)$ for $K \rightarrow \infty$ : since, again, this is the transform of the real potential, it cannot depend on the atomic mass. Our final choice is therefore represented by $\Phi_{l}(K)=A$, with $A$ independent on $K, m$ and $T$. The corresponding form of $\varphi_{l}(\boldsymbol{r})$ is a delta function, namely $A \delta(\boldsymbol{r})$. The extension of this potential to the case of light atoms is easily justifiable. In fact it describes an interaction between pointlike particles and therefore corresponds to an approximation which, in principle, should work even better for atoms with smaller rigid sphere diameters, like Li (see Table 1). For these atoms, the expression of $l$ will be provided by eq. (21).

Since the range of the interaction described by a delta function is vanishingly small, we note the full consistency of this function with the conditions required for the validity of the kinetic formula (19). We also note that, in such a potential, the dependence of the pair interaction on the chemical nature of the atoms is entirely committed to the constant $A$, which in principle depends on $Z_{e}$, the total number of atomic electrons, but is independent of $\Delta$.

For $\Phi_{l}(K)=A$ one finds $I=(\pi+2) A^{2} / 8$, so that

$$
l_{\mathrm{o}}^{-1}=4.04 \cdot 10^{7} v^{-1 / 3} Z_{e}^{2} Z_{V}^{4 / 3}\left(A / v \varepsilon_{\mathrm{F}}\right)^{2}
$$

Eqs. (21)-(23) are equivalent to the expression given in I, namely

$$
l^{-1}=m^{2} A^{2} /\left(7.678 v \hbar^{4}\right)
$$

which in fact can be directly obtained from the expression of $\mathcal{S}$ by the help of the mentioned value of $I$. Eqs.(21)-(23), however, are more interesting than (24): they emphasize the fact that the only way to appreciate the mass dependence of $l$ is through the isotope effect, that is through a change of the parameter $\Delta$. In principle, the experimental test of this dependence requires to change $m$ at constant $A$, and 
this is clearly possible only if different isotopes of the same element are used. In the absence of isotopes, the mass dependence of $l$, as indicated by eq. (24), is misleading: in fact in such a case, following the procedure used to arrive at eq. (23), we can always express $m$ in terms of $Z_{e}$ and interpret $l$ as depending only on the physical properties of the atomic electron clouds $\left(Z_{e}, Z_{V}, \varepsilon_{\mathrm{F}}, A\right)$, that is on the parameters determining the structure, rather than the dynamical behaviour of the liquid. This is precisely what the formula $l=\xi R_{A}$ suggests for heavy atoms, where in fact the isotope effect is negligible.

As a final comment to this section, we note that the choice $\Phi_{l}(K)=A$ allows eq. (17) to be written in the form $\Phi^{2}(K)=\chi_{c}(K)+A^{2}$. Inserting into eq. (24) the expected order of magnitude of $l$ (some $10^{-1} \AA$ ) we deduce for $A$ a value which is about three orders of magnitude lower than $\Phi(0)$, as given by eq. (18). This means that $\chi_{c}(0)$ is enormously higher than $A^{2}$, so that $\chi_{c}(0) \cong \Phi^{2}(0)$ and eq. (24) can be written in the form

$$
\Phi^{2}(K)=\Phi^{2}(0) Y\left(x^{\prime}\right)+A^{2}
$$

where $Y\left(x^{\prime}\right)$ is a short range function of the dimensionless variable $x^{\prime}=K / K_{m}$, starting from $Y(0)=1$ and vanishing for $x^{\prime}>1$.

A decomposition analogous to (25) was already introduced in I, however by expressing $Y$ in terms of the dimensionless variable $x=K \sigma, \sigma$ being the rigid sphere diameter. As a consequence, in order to compare the results of the present theory with those obtained in the previous formulation, it will be sufficient to substitute $K_{m}$ by $1 / \sigma$. In I it was only argued that the cut off abscissa $x_{c}$, measuring the range of $Y$ on the $x$ axis, must be significantly smaller than $2 \pi$. This conclusion was sufficient to discuss the mass dependence of the self-diffusivity, but overlooked the physical meaning of $x_{c}$, namely its relation with the collective modes, which becomes essential to understand the influence of temperature on the diffusion process.

\section{5 - Expression of the diffusion coefficients.}

The first step consists in the evaluation of the double sum appearing on the right hand side of eq. (7). For a given $\boldsymbol{q}$, it is convenient to transform the sum over $\boldsymbol{q}^{\prime}$ into a sum over all the vectors $\boldsymbol{K}$. The resulting double sum over $\boldsymbol{q}$ and $\boldsymbol{K}$ can then be easily transformed into a multiple integral. To this purpose one first performs the integrations over the polar angles $\theta, \phi$ defining the orientation of $\boldsymbol{K}$ in a polar reference frame where $\theta$ is the angle between $\boldsymbol{K}$ and $\boldsymbol{q}$ and $\phi=0$ corresponds to $\boldsymbol{K}$ lying in the plane formed by $\boldsymbol{q}$ and $\boldsymbol{U}$. The result of the integration depends on the angle $\theta^{\prime}$ between $\boldsymbol{q}$ and $\boldsymbol{U}$ : at this point one can integrate over the directions of vector $\boldsymbol{q}$, specified by angles $\theta^{\prime}$ and $\phi^{\prime}$ in a new reference frame with polar axis along $\boldsymbol{U}$. The integral under study turns out to be of the form

$$
\begin{gathered}
\mathcal{L}=\int_{0}^{\infty} q^{2} \mathrm{~d} q \int_{0}^{\pi} \sin \theta^{\prime} \mathrm{d} \theta^{\prime} \int_{0}^{2 \pi} \mathrm{d} \phi^{\prime} \int_{0}^{\infty} \mathrm{d} K \int_{0}^{\pi} \sin \theta \mathrm{d} \theta \int_{0}^{2 \pi} \mathrm{d} \phi \mathcal{F}(K, q, \cos \theta) \\
{\left[\alpha(\boldsymbol{K} \cdot \boldsymbol{U})+\beta^{\prime}|\boldsymbol{q}+\boldsymbol{K}|^{3}(\boldsymbol{q}+\boldsymbol{K}) \cdot \boldsymbol{U}-\beta^{\prime} q^{3}(\boldsymbol{q} \cdot \boldsymbol{U})\right]^{2}}
\end{gathered}
$$


where $\beta^{\prime}=\lambda_{a}^{3 / 2} \beta$.

Performing the integrations over $\phi, \phi^{\prime}, \theta^{\prime}$ and putting $\cos \theta=\xi$ one easily finds

$$
\begin{gathered}
\mathcal{L}=\left(8 \pi^{2} / 3\right) \int_{0}^{\infty} \mathrm{d} K \int_{0}^{\infty} q^{2} \mathrm{~d} q \int_{-1}^{1} \mathrm{~d} \xi \mathcal{F}(K, q, \xi)\left[\left(\alpha+\beta^{\prime} M\right)^{2} K^{2}+\beta^{\prime 2} q^{2}\left(M-q^{3}\right)^{2}\right. \\
\left.+2 \beta^{\prime} q K \xi\left(\alpha+\beta^{\prime} M\right)\left(M-q^{3}\right)\right]
\end{gathered}
$$

where $M=\left(q^{2}+K^{2}+2 q K \xi\right)^{3 / 2}$. By the help of this result, going to the usual dimensionless variables $y$ and $u$ and recalling the definitions (11) and (12) one obtains

$$
\begin{aligned}
& \Sigma_{q, q^{\prime}}\left(\psi_{\boldsymbol{q}}-\psi_{q^{\prime}}\right)^{2} S\left(\boldsymbol{q}, \boldsymbol{q}^{\prime}\right)=\Omega^{2}(2 \pi)^{-6} \mathcal{R} \int_{0}^{\infty} y^{3} \Phi^{2}\left(\lambda_{a}{ }^{-1 / 2} y\right) \mathrm{d} y \int_{0}^{\infty} u^{2} \exp \left(-u^{2}\right) \mathrm{d} u \int_{-1}^{1} \mathrm{~d} \xi \\
& {\left[\left(\alpha+\beta t^{3}\right)^{2}+\beta^{2} u^{2} y^{-2}\left(t^{3}-u^{3}\right)^{2}+2 \beta u y^{-1} \xi\left(\alpha+\beta t^{3}\right)\left(t^{3}-u^{3}\right)\right] \exp (-\mathcal{K})}
\end{aligned}
$$

where $t$ is defined by eq. (11) and

$$
\mathcal{R}=\left(16 \pi^{3} / 3\right)\left(m_{b} / 2 \pi k_{\mathrm{B}} T\right)^{1 / 2}\left(v \hbar^{2} \lambda_{a}^{7 / 2}\right)^{-1}
$$

The first and second term on the right hand side of eq. (25) correspond to two distinct contributions, $\mathcal{A}$ and $\mathcal{B}$, respectively, to the integral (28). Since $Y\left(x^{\prime}\right)$ in (25) is negligible for $x^{\prime}>1$ and $x^{\prime}$ is linked to the variable $y$ through the relation $y$ $=K_{m} \lambda_{a}^{1 / 2} x^{\prime}$, where $K_{m} \lambda_{a}^{1 / 2}$ is much smaller than $Q_{\mathrm{D}} \lambda_{a}^{1 / 2}=0.11$ for $\mathrm{Li}^{7}$ at the melting point, only the limit for $y \rightarrow 0$ of the square bracket of (28) is actually of interest for the evaluation of $\mathcal{A}$. In this way, putting

$$
\begin{aligned}
& \mathcal{J}=\int_{0}^{\infty} x^{\prime 3} Y\left(x^{\prime}\right) \mathrm{d} x^{\prime} \\
& \mathcal{H}=K_{m}{ }^{4} g \Phi^{2}(0)
\end{aligned}
$$

we find

$$
\begin{gathered}
\mathcal{A}=\Omega^{2}(2 \pi)^{-6} \mathcal{R} \mathcal{H} \lambda_{a}^{2} \int_{0}^{\infty} u^{2} \exp \left(-u^{2}\right) \mathrm{d} u \int_{-1}^{1}\left[\left(\alpha+\beta u^{3}\right)^{2}+9 \beta^{2} u^{6} \xi^{2}+\right. \\
\left.6 \beta\left(\alpha+\beta u^{3}\right) u^{3} \xi^{2}\right] \exp \left(-\mu u^{2} \xi^{2}\right) \mathrm{d} \xi
\end{gathered}
$$

Introducing the dimensionless functions of $\mu$ 


$$
\begin{aligned}
& R_{1}=\int_{0}^{\infty} u^{2} \exp \left(-u^{2}\right) \mathrm{d} u \int_{-1}^{1} \exp \left(-\mu u^{2} \xi^{2}\right) \mathrm{d} \xi \\
& R_{2}=\int_{0}^{\infty} u^{5} \exp \left(-u^{2}\right) \mathrm{d} u \int_{-1}^{1}\left(1+3 \xi^{2}\right) \exp \left(-\mu u^{2} \xi^{2}\right) \mathrm{d} \xi \\
& R_{3}=\int_{0}^{\infty} u^{8} \exp \left(-u^{2}\right) \mathrm{d} u \int_{-1}^{1}\left(1+15 \xi^{2}\right) \exp \left(-\mu u^{2} \xi^{2}\right) \mathrm{d} \xi
\end{aligned}
$$

we can rewrite $\mathcal{A}$ in the simple form

$$
\mathcal{A}=\Omega^{2}(2 \pi)^{-6} \mathcal{R} \lambda_{a}^{2}\left(\alpha^{2} R_{1}+2 \alpha \beta R_{2}+\beta^{2} R_{3}\right)
$$

In a similar way, introducing the functions of $\mu$

$$
\begin{aligned}
& S_{1}=\int_{0}^{\infty} y^{3} \mathrm{~d} y \int_{0}^{\infty} u^{2} \exp \left(-u^{2}\right) \mathrm{d} u \int_{-1}^{1} \exp (-\mathcal{K}) \mathrm{d} \xi \\
& S_{2}=\int_{0}^{\infty} y^{3} \mathrm{~d} y \int_{0}^{\infty} u^{2} \exp \left(-u^{2}\right) \mathrm{d} u \int_{-1}^{1}\left[t^{3}+u y^{-1} \xi\left(t^{3}-u^{3}\right)\right] \exp (-\mathcal{K}) \mathrm{d} \xi \\
& S_{3}=\int_{0}^{\infty} y^{3} \mathrm{~d} y \int_{0}^{\infty} u^{2} \exp \left(-u^{2}\right) \mathrm{d} u \int_{-1}^{1}\left[t^{6}+u^{2} y^{-2}\left(t^{3}-u^{3}\right)^{2}+\right. \\
& \left.2 u y^{-1} \xi t^{3}\left(t^{3}-u^{3}\right)\right] \exp (-\mathcal{K}) \mathrm{d} \xi
\end{aligned}
$$

we obtain

$$
\mathcal{B}=\Omega^{2}(2 \pi)^{-6} \mathcal{R} A^{2}\left(\alpha^{2} S_{1}+2 \alpha \beta S_{2}+\beta^{2} S_{3}\right)
$$

Functions $R_{j}$ and $S_{j}$ have been determined for the values of $\mu$ of interest for liquid lithium, namely $1,7 / 6$ and 6/7: the results are given by Table 2 .

The evaluation of the sum appearing on the left hand side of eq. (7) is easier. The result is

$$
\Sigma_{\boldsymbol{q}}\left(\boldsymbol{V}_{\boldsymbol{q}} \cdot \boldsymbol{U}\right) \psi_{\boldsymbol{q}} \exp \left(-\lambda_{a} q^{2}\right)=\Omega(2 \pi)^{-3} \pi^{3 / 2} \hbar\left(2 m_{a} \lambda_{a}^{5 / 2}\right)^{-1}\left(\alpha+8 \pi^{-1 / 2} \beta\right)
$$

Eq. (7) can then be written in the form

$$
\begin{gathered}
-X\left(\lambda_{a} m_{a}\right)^{-1} \hbar\left(\alpha+8 \pi^{-1 / 2} \beta\right)=(2 \pi)^{-3} \mathcal{R} \lambda_{a}^{2}\left[\left(R_{1}+\rho_{a} S_{1}\right) \alpha^{2}+\right. \\
\left.2\left(R_{2}+\rho_{a} S_{2}\right) \alpha \beta+\left(R_{3}+\rho_{a} S_{3}\right) \beta^{2}\right]
\end{gathered}
$$


where

$$
\rho_{a}=A^{2} /\left(\lambda_{a}^{2} \mathcal{H}\right)=4 A^{2} m_{a}^{2}\left(k_{\mathrm{B}} T\right)^{2} /\left(\hbar^{4} \mathcal{H}\right)
$$

and, according to the variational principle, $\alpha$ and $\beta$ have to be determined in such a way that the right hand side of eq. (42) is a maximum, under the constraint represented by eq. (42) itself. Using the standard procedure of Lagrange multipliers we find

$$
\alpha=-(2 \pi)^{3} \hbar X\left(\mathcal{R} m_{a} \lambda_{a}{ }^{3}\right)^{-1}\left(1+8 \pi^{-1 / 2} f\right) / Z
$$

and $\beta=f \alpha$, where

$$
\begin{gathered}
Z=R_{1}+\rho_{a} S_{1}+2\left(R_{2}+\rho_{a} S_{2}\right) f+\left(R_{3}+\rho_{a} S_{3}\right) f^{2} \\
f=\left[8 \pi^{-1 / 2}\left(R_{1}+\rho_{a} S_{1}\right)-\left(R_{2}+\rho_{a} S_{2}\right)\right] /\left[R_{3}+\rho_{a} S_{3}-8 \pi^{-1 / 2}\left(R_{2}+\rho_{a} S_{2}\right)\right]
\end{gathered}
$$

In this way the trial function (15) takes the form $\alpha\left(1+f u^{3}\right)(\boldsymbol{q} \cdot \boldsymbol{U})$ : when compared to (8), this implies that $G(u)$ is proportional to $\left(1+f u^{3}\right)\left(1+8 \pi^{-1 / 2} f\right) / Z$. From Table 2 we deduce that $f$ turns out to be positive for $\rho_{a}<\rho^{*}$, where $\rho^{*}$ is $1.35,1.15$ and 1.59 for $\mu=1, \mu=7 / 6$ and $\mu=6 / 7$, respectively: consequently, if $\rho_{a}$ satisfies the above condition, also $G(u)$ is positive on the whole axis of its variable. This is a proof of the reliability of the variational procedure when applied to liquid lithium, where in fact $\rho_{a}$ will always be found lower than $\rho^{*}$ (see sect.6).

It is now easy to evaluate the current density (14) and deduce for the diffusion coefficient the expression

$$
D^{a / b}=3 \pi^{2} \sqrt{2} v\left(k_{\mathrm{B}} T\right)^{5 / 2} m_{b}^{-1 / 2} \mathcal{H}^{-1} W\left(\mu ; \rho_{a}\right)
$$

where the function $W$ is defined as

$$
W\left(\mu ; \rho_{a}\right)=\left(1+8 \pi^{-1 / 2} f\right)^{2} / Z
$$

The above expression is expected to be coincident with the one correspondingly obtained in I and II when the term $\beta q^{3}$ in the trial function is neglected. In fact in such a case $f, R_{2}, R_{3}, S_{2}, S_{3}$ are all zero, so that, from the analytical expressions of $R_{1}$ and $S_{1}$, namely

$$
\begin{aligned}
& R_{1}=\left(\pi^{1 / 2} / 2\right)(1+\mu)^{-1 / 2} \\
& S_{1}=4 \pi^{1 / 2}(1+\mu)^{-1 / 2}\left(1+\mu^{-1}\right)^{-2}
\end{aligned}
$$

we obtain

$$
D^{a / b}=6\left(2 \pi^{3}\right)^{1 / 2} v\left(k_{\mathrm{B}} T\right)^{5 / 2} M_{a b}^{-1 / 2} \mathcal{H}^{-1}\left[1+\varepsilon M_{a b}{ }^{2}\left(k_{\mathrm{B}} T\right)^{2}\right]^{-1}
$$

where $M_{a b}=m_{a} m_{b} /\left(m_{a}+m_{b}\right)$ and 


$$
\varepsilon=32 A^{2} /\left(\hbar^{4} \mathcal{H}\right)
$$

Eq. (51) becomes precisely the result obtained in I and II if $K_{m}$, as explained in sect. 4 , is roughly identified with $1 / \sigma$.

\section{6 - Mass dependence of self-diffusion.}

Self-diffusion corresponds to the case in which the solute and solvent atoms have the same mass, $m_{a}=m_{b}=m_{s}$. For reasons that will be clarified later, it is convenient to express the self-diffusion coefficient $D^{s / s}$ in terms of its value referring to $\rho_{s}=0$, that is of

$$
D_{\mathrm{o}}^{s / s}=1.896 \cdot 3 \pi^{2} \sqrt{2} v\left(k_{\mathrm{B}} T\right)^{5 / 2} \mathcal{H}^{-1} m_{s}^{-1 / 2}
$$

where the numerical coefficient $1.896=W(1 ; 0)$ derives from the first column of Table 2. From eqs. (47) and (53) we obtain

$$
D^{s / s}=0.527 W\left(1 ; \rho_{s}\right) D_{\mathrm{o}}^{s / s}
$$

To compare our result with that of the classical theory of diffusion, as developed for a liquid of rigid spheres, let us consider the Enskog expression of the self-diffusion coefficient $[4,5,13,14]$

$$
\left(D^{s / s}\right)_{\mathrm{r} . \mathrm{s} .}=\left(3 v / 8 \sigma^{2}\right)\left(k_{\mathrm{B}} T / \pi m_{s}\right)^{1 / 2}(1-\eta)^{3}(1-\eta / 2)^{-1}
$$

where $\eta=\pi \sigma^{3} / 6 v$ is the packing fraction. This formula is obtained when the memory function which describes the frictional force in the Langevin equation is written in the form $\Gamma_{\mathrm{o}} \delta\left(t-t^{\prime}\right)$. The approximation is reliable only if one assumes a very long length of free motion (Shimoji [14] , p. 187): in more precise terms, the mean free path must be large with respect to $\sigma$. Thus eq. (55) has to be compared with the expression corresponding to eq. (54) in the limit $l \rightarrow \infty$. Since, according to eqs. (24) and (43), $l$ is inversely proportional to $\rho_{s}$, this means that we must precisely compare eq. (55) with eq. (53).

The physical meaning of such a comparison is clarified by the fact that eq. (55), being strictly related to the condition $l \rightarrow \infty$, cannot take into account large angle scattering processes, which we have shown to be responsible for the finite value of the mean free path. Thus eq. (55), neglecting the so called back scattering effect, corresponds to an approximate description where the main role is played by low angle scattering processes. These are precisely, in our description, the only processes allowed by the collective interaction, that is the only processes contributing to $D_{\mathrm{o}}^{s / s}$ : therefore, the comparison between eq. (53) and eq. (55) becomes justified.

In comparing the two temperature dependences, which are quite different, we point out that the $T^{1 / 2}$ law predicted by eq. (55) is strongly in contrast with the FMS experimental data. This is not surprising, because eq. (55) disregards correlations between successive collisions, i. e. events involving more than two particles. Since the weight of the correction due to these correlations is expected to increase with 
temperature, such an equation is expected to be reliable only at low temperatures. The lowest temperature at disposal of the liquid corresponds to the melting point $T_{m}$ and there are strong reasons to believe that at this temperature the correction is negligible. In fact from eq. (55), using the data of Table 1 , we find for sodium $\left(T_{m}=373 \mathrm{~K}\right)$ the value $6.13 \cdot 10^{-9} \mathrm{~m}^{2} / \mathrm{s}$. This is in full agreement with the value calculated by FMS [4] through a model where the above correlations were explicitly introduced: on the contrary, strong discrepancies are found between the predictions of this model and eq. (55) at higher temperatures. Starting from these considerations and from the well known reliability of the rigid sphere model in the description of many structure and transport properties of liquid metals, we will consider $D_{0}^{s / s}$ to be well represented at the melting point by the numerical value predictable throug eq. (55). The identification of eqs. (53) and (55) at $T=T_{m}$ is equivalent to assuming that these equations, based on opposite approximations of the pair interaction (soft and hard pair potential, respectively), are representative of two complementary and equally reliable models in the theory of diffusion.

A first consequence of this identification is the possibility of obtaining information on $\mathcal{H}$. Since the factor $m_{s}^{-1 / 2}$ appears in both the equations, one concludes that $\mathcal{H}$ is independent of the atomic mass. There is a more general justification of this result. Let us note, in fact, that the presence of $\hbar$ in eq. (47) is confined to the parameter $\rho_{a}$ defined by eq. (43), so that in our description the classical result corresponds to the limit for $\rho_{s} \rightarrow 0$, that is to $D_{\mathrm{o}}{ }^{s / s}$. Since any classical theory of diffusion is expected to predict the $m_{s}^{-1 / 2}$ law (see FMS, [4]), it follows from expression (53) of $D_{\mathrm{o}}^{s / s}$ that this is possible only if $\mathcal{H}$ is mass independent. This property is consistent with the fact that all the parameters entering the definition of $\mathcal{H}$ are related to the features of the pseudopotential, which is expected to be mass-independent: it will be helpful to discuss the temperature behaviour of the self-diffusion coefficients (sect. 7).

A second consequence of the identification of eqs. (53) and (55) at $T=T_{m}$ is the acquisition of the numerical value of the product $K_{m}^{4}{ }^{4} \mathrm{~g}$. For $\mathrm{Li}^{7}$ at the melting point, using eq. (18) for $\Phi(0)$ and the values of $a_{0}, \eta, v$ and $\sigma$ given in Table 1, we obtain $K_{m}{ }^{4} g=2.19 \cdot 10^{-2} \AA^{-4}$. Since the leading contribution to the integral (30) is expected to come from values of $K$ lower than $K_{m}$, that is from $x^{\prime}<1$, we can approximately take for $Y\left(x^{\prime}\right)$ a step function, equal to unity in this range and zero outside. This means $g=1 / 4$ and $K_{m}=0.54 \AA^{-1}$. As expected, $K_{m}$ turns out to be much lower than the Debye radius, which in fact is $1.38 \AA^{-1}$. A similar conclusion is reached for sodium and potassium, as shown by the values of the ratio $K_{m} / Q_{\mathrm{D}}$ appearing in the above Table. Since the number of the equilibrium phonon modes is proportional to $K_{m}{ }^{3}$, we deduce that these modes represent a fraction of the order of a few per cents of the total number of degrees of freedom.

A third consequence consists in the determination of the value of $\rho_{s}$ for $\mathrm{Li}^{7}$ at the melting point, hereafter denoted by $\rho_{7}\left(T_{m}\right)$, as deducible by inserting into eq. (54) the corresponding experimental value of $D^{7 / 7}$. In order to compare the present formulation with the theory developed in I, let us take for $D^{7 / 7}$ the value used in the above paper, namely $6.1 \cdot 10^{-9} \mathrm{~m}^{2} \mathrm{~s}^{-1}$. In this case we obtain $\rho_{7}\left(T_{m}\right)=0.347$. Owing to eqs. (24), (31) and (43), the mean free path is now obtained by the formula

$$
l=30.71\left(K_{m}{ }^{4} g v \rho_{s}\right)^{-1}\left(a_{0}{ }^{-1}-1\right)^{-2}
$$


which for $\rho_{s}=0.347$ provides the numerical value $0.182 \AA$. This is in good agreement with the result obtained in I, namely $0.157 \AA$.

It is now easy to arrive at the following expression of the ratio between the selfdiffusion coefficients of $\mathrm{Li}^{6}$ and $\mathrm{Li}^{7}$ at the melting point:

$$
\left(D^{6 / 6} / D^{7 / 7}\right)_{\text {m.p. }}=\left(m_{7} / m_{6}\right)^{1 / 2} W\left[1 ;\left(m_{6} / m_{7}\right)^{2} \rho_{7}\left(T_{m}\right)\right] / W\left[1 ; \rho_{7}\left(T_{m}\right)\right]
$$

Introducing into eq. (57) the value of $\rho_{7}\left(T_{m}\right)$ previously determined and using Table 1 we deduce $D^{6 / 6} / D^{7 / 7}=1.230$. The relevance of this result lies in the excellent agreement with the ratio 1.24 , as obtained in I through the simple choice $G(u)=$ constant. This shows on one hand the convergence of the variational method of solution, on the other hand the full reliability of the trial function used in I.

The use of the experimental value of $D^{7 / 7}$ given by FMS for the melting point, namely $5.64 \cdot 10^{-9} \mathrm{~m}^{2} \mathrm{~s}^{-1}$, provides through eq. (54) $\rho_{7}\left(T_{m}\right)=0.410$ and consequently a mean free path of $0.154 \AA$. The corresponding ratio (57) turns out to be 1.243 . This is in full agreement with the ratio measured by the same authors, that is 1.25.

For the sake of completeness, we give in Table 1 the mean free paths of $\mathrm{Li}, \mathrm{Na}$ and $\mathrm{K}$ at the melting point, as predicted through eq. (56) and the identification of (53) and (55). As shown by the last column, $l$ lies precisely between $0.1 R_{\mathrm{A}}$ and $0.2 R_{\mathrm{A}}$, in full agreement with our expectations. Such a result has to be considered a strong support in favour of the above identification.

\section{7 - Temperature dependence of the self-diffusion coefficients.}

We obtain, from eq. (43),

$$
\rho_{\alpha}(T)=\left(m_{\alpha} / m_{7}\right)^{2}\left(T / T_{m}\right)^{2}\left[\mathcal{H}\left(T_{m}\right) / \mathcal{H}(T)\right] \rho_{7}\left(T_{m}\right)
$$

where $\alpha$ is an index running over the two values 6 and 7 . On the other hand, eqs. (53) and (54) provide

$$
\begin{aligned}
& D^{\alpha / \alpha}(T) / D^{\alpha / \alpha}\left(T_{m}\right)= \\
& \left\{W\left[1 ; \rho_{\alpha}(T)\right] / W\left[1 ; \rho_{\alpha}\left(T_{m}\right)\right]\right\}\left(T / T_{m}\right)^{5 / 2}\left[\mathcal{H}\left(T_{m}\right) / \mathcal{H}(T)\right]\left[v(T) / v\left(T_{m}\right)\right]
\end{aligned}
$$

From eqs. (58) and (59), written for $\alpha=7$, by the use of the previously determined value of $\rho_{7}\left(T_{m}\right)$ and of the experimental values of the ratio $D^{7 / 7}(T) / D^{7 / 7}\left(T_{m}\right)$ deducible from FMS, it is possible to obtain the behaviour of $\mathcal{H}(T) / \mathcal{H}\left(T_{m}\right)$ as a function of temperature. Such a behaviour (not explicitly reported here) turns out to be a decreasing function of $T$. We find therefore a result fully consistent with the discussion contained in sect. 3 , where the long wavelength part of the pseudopotential (contained in $\mathcal{H}(T)$ ) was shown to be connected with the collective modes present in the liquid: these require correlation effects, which are expected to be weakened if we rise $T$, because in this way we are going towards a gas like condition: in particular, an 
increase of temperature determines a reduction of the total number of phonon modes, that is of $K_{m}$.

To these qualitative arguments, which play in favour of the self-consistency of the whole description, we can now add an important quantitative check, as provided by eqs. (58) and (59) for $\alpha=6$. In fact, owing to the previous determination of function $\mathcal{H}(T) / \mathcal{H}\left(T_{m}\right)$ and to its independence of the atomic mass (see sect. 6), these equations are able to predict the temperature dependence of the ratio $D^{6 / 6}(T) / D^{6 / 6}\left(T_{m}\right)$, which can be finally compared with experiment.

FMS expressed the results of their measurements in terms of the exponent $n$ in the power law $D^{\alpha / \alpha}=C T^{n}$, by which they empirically represented the temperature dependence of the self-diffusion coefficient in the interval between $454 \mathrm{~K}$ and 580 $\mathrm{K}$. For $\mathrm{Li}^{7}$ they found $n=2.37$, leading to a ratio $D^{7 / 7}\left(T_{1}\right) / D^{7 / 7}\left(T_{m}\right)=1.787$ at $T_{1}=$ $580 \mathrm{~K}$. The corresponding ratio $\mathcal{H}\left(T_{1}\right) / \mathcal{H}\left(T_{m}\right)$ is deducible from the solution of eqs. (58) and (59) written for $\alpha=7$ and the use of the thermal expansion coefficient (1.9 . $10^{-4} \mathrm{~K}^{-1}$, [15] ) for the evaluation of the atomic volume ratio: we obtain $\mathcal{H}\left(T_{1}\right) / \mathcal{H}\left(T_{m}\right)$ $=0.548$. Substituting this value into the same equations written for $\alpha=6$ provides the numerical result $D^{6 / 6}\left(T_{1}\right) / D^{6 / 6}\left(T_{m}\right)=1.917$. This corresponds to representing $D^{6 / 6}(T)$ through a power law defined by the exponent $n=2.66$. Such a result is in remarkable agreement with the experimental value (2.68) quoted by the same authors for $\mathrm{Li}^{6}$ : it shows, therefore, that the present theory explains the temperature dependence of the dynamic isotope effect (defined, according to Buchhauser et al. [5], by the ratio $D^{6 / 6} / D^{7 / 7}$ ).

The above important check can be considered as independent of the rigid sphere model: in fact it can be achieved without resorting to the identification $D_{\mathrm{o}}^{s / s}=\left(D^{s / s}\right)_{\text {r.s. }}$ at the melting point. To this purpose, in applying eqs. (58)-(59), it is sufficient to use for $\rho_{7}\left(T_{m}\right)$ the value provided by eq. (57) when the left hand side of this equation is substituted by the experimental ratio 1.25 . In such a procedure, one renounces any prediction concerning the ratio $D^{6 / 6} / D^{7 / 7}$ but explains, independently of any model, the stronger temperature dependence of the self-diffusion coefficient of $\mathrm{Li}^{6}$ with respect to that of $\mathrm{Li}^{7}$. The numerical results cannot be significantly different from those previously obtained, because the ratio 1.243 predicted through the rigid sphere model is not distinguishable, within the experimental uncertainties, from the ratio measured by FMS.

Owing to eq. (58), the value 0.548 previously found for the ratio $\mathcal{H}\left(T_{1}\right) / \mathcal{H}\left(T_{m}\right)$ at $580 \mathrm{~K}$ corresponds to $\rho_{7}\left(T_{m}\right)=1.22$. This is still lower than $\rho^{*}=1.35$, the upper limit of validity for the trial function (15), see sect. 5: therefore the interpretation of the FMS data in the framework of our variational procedure is fully consistent. We point out, however, that no appreciable error would be introduced by the application of the same procedure to higher temperatures, corresponding to values of $\rho_{s}$ higher than $\rho^{*}$. In fact, in the interval $\rho^{*} \leq \rho_{s} \leq \infty, f$ is negative but very small, monotonically decreasing from zero to -0.0203 : this means that in such an interval function $1+f u^{3}$ and consequently $G(u)$, becomes negative only for $u>\left|f\left(\rho_{s}\right)\right|^{-1 / 3} \geq 3.67$. Because of the presence of $\exp \left(-u^{2}\right)$ in eqs. (33)-(35) and (37)-(39), the inconsistency of a negative value of $G(u)$ is therefore confined to a region of the $u$ axis which is expected to provide a negligible contribution to the integral (28): for this reason it can be completely disregarded. 
It is interesting to investigate the high temperature behaviour of expression (54) in the limit in which any correlation is lost, as a consequence of the fact that the system has reached a gas like condition. In this case $a_{0} \sim 1$ so that, owing to eqs. (18) and (31), $\mathcal{H}(T)$ vanishes while, owing to eq. (43), $\rho_{s} \propto T^{2} / \mathcal{H}$ is very large: we have simply to perform the limit of eq. (54) for $\rho_{s} \rightarrow \infty$. Again we neglect the small error deriving from the use of (15) in a case where $\rho_{s}>\rho^{*}$ and find

$$
D^{s / s} \sim 0.805\left(3 \pi^{2} / 4\right)\left(2 k_{\mathrm{B}} T\right)^{1 / 2} v \hbar^{4} A^{-2} m_{s}^{-5 / 2}
$$

Because of eq. (24), eq. (60) can also be written

$$
D^{s / s} \sim \gamma\left(k_{\mathrm{B}} T / m_{s}\right)^{1 / 2} l
$$

with $\gamma=1.10$.

The form of eq. (61) is well known to be correct for the gaseous state. On the other hand, a classical argument used in I (see Appendix D of the above paper) provides the expression $\gamma=(1 / 6)(8 / \pi)^{1 / 2} \xi$, where $\left.\xi=\left\langle l^{2}\right\rangle /<l\right\rangle^{2}$. Assuming for a rarefied gas $\xi=2$ (see Faber [13], p.160) we obtain $\gamma=0.53$. The discrepancy is easily understandable because the value 1.10 was deduced from our pseudopotential formulation, which is presumed to be reliable only for metallic densities. For a gas with such a high density, it is not surprising that the value of $\xi$ be at variance with respect to that corresponding to the low density limit.

\section{8 - Interdiffusion between $\mathrm{Li}^{6}$ and $\mathrm{Li}^{7}$.}

By the help of eq. (47) it is also possible to discuss the diffusion of $\mathrm{Li}^{6}$ moving through pure $\mathrm{Li}^{7}$ and of $\mathrm{Li}^{7}$ moving through pure $\mathrm{Li}^{6}$. One easily obtains, for the ratio between the corresponding diffusion coefficients,

$$
D^{6 / 7} / D^{7 / 6}=\left(m_{6} / m_{7}\right)^{1 / 2} W\left[7 / 6 ;\left(m_{6} / m_{7}\right)^{2} \rho_{7}\right] / W\left[6 / 7 ; \rho_{7}\right]
$$

At the melting point we deduced $\rho_{7}=0.410$. Substituting this value into the above equation, by the help of Table 2 , gives $D^{6 / 7} / D^{7 / 6}=1.01$.

FMS determined at the melting point, in a liquid mixture of $\mathrm{Li}^{6}$ and $\mathrm{Li}^{7}$, the diffusivities of both the isotopes as a function of $x_{7}$, the mole fraction of $\mathrm{Li}^{7}$ in the mixture. When the two corresponding curves are extrapolated at $x_{7}=1$ and $x_{7}=0$, respectively, one finds, in units $10^{-5} \mathrm{~cm}^{2} / \mathrm{s}, D^{6 / 7}=6.2 \pm 0.3$ and $D^{7 / 6}=6.5 \pm 0.3$, where \pm 0.3 is deduced from the length of the uncertainty bars appearing in the figures of the quoted papers. The two results are perfectly consistent with the theoretical prediction of eq. (62), because they turn out to be substantially equal within the experimental uncertainties. It is worth noting that the use of the simple trial function adopted in I gave the rigorous equality of $D^{6 / 7}$ and $D^{7 / 6}$. This represents a further proof of the reliability of such a function as an approximate solution of the Boltzmann equation.

\section{9 - Conclusions.}


The consistency of the present theory with the FMS experimental data can be summarized through the following points:

$a$ ) the theory explains the experimental ratio of the self-diffusivities of $\mathrm{Li}^{6}$ and $\mathrm{Li}^{7}$;

$b$ ) it explains the stronger temperature dependence of the self-diffusivity of $\mathrm{Li}^{6}$ with respect to that of $\mathrm{Li}^{7}$;

$c$ ) it explains, within the experimental uncertainties, the closeness of the two interdiffusion coefficients $D^{6 / 7}$ and $D^{7 / 6}$.

Elements of internal consistency of the theory are

$d$ ) the convergence of the variational procedure adopted to solve the transport equation, as shown by the agreement between the results of the present paper and those previously obtained through the use of a simpler form of trial function;

$e$ ) the small fraction of phonon modes, as deduced from the self-diffusivities at the melting point, in full agreement with the basic assumptions on which the theory is founded;

$f$ ) the correct order of magnitude predicted for the atomic mean free paths.

With reference to point $d$ ) it has to be noted that eq. (51), corresponding to the trial function adopted in I, can correctly be used instead of eq. (47) for an approximate but satisfactory description of diffusion phenomena.

The present theory cannot be considered a first principle approach to the description of diffusion in metals, inasmuch as it uses a model potential whose behaviour, in the interval of the $K$ axis of interest for collective modes, is not perfectly known. As a consequence of this lack of information, we cannot predict the temperature dependence of both the self-diffusivities of $\mathrm{Li}^{6}$ and $\mathrm{Li}^{7}$ : we can only predict $D^{6 / 6}(T)$ when $D^{7 / 7}(T)$ is known, and viceversa. In spite of these limitations, the remarkable agreement between theoretical and experimental values of all the quantities concerning points $a), b$ ) and $c$ ) is a clear proof of the reliability of the pseudopotential formulation: we believe that this can be considered as the simplest tool at our disposal to understand the isotope effects observed in diffusion processes of liquid metals.

\section{Appendix A.}

Let us write the matrix element for a process in which a solute atom with wavevector $\boldsymbol{q}$ is scattered into a state with wavevector $\boldsymbol{q}^{\prime}=\boldsymbol{q}+\boldsymbol{K}$, owing to its interaction with a dynamical configuration of the host liquid characterized by atoms with wavevectors $\boldsymbol{Q}_{1}, \ldots \boldsymbol{Q}_{j}, \ldots \boldsymbol{Q}_{N}$. One has

$$
<\Psi^{\prime}\left|H^{\prime}\right| \Psi>=\int \mathrm{d}^{3} r \int \mathrm{d}^{3} R_{1} \ldots \int \mathrm{d}^{3} R_{N} \Psi^{*} \Sigma_{j} \varphi\left(\boldsymbol{r}-\boldsymbol{R}_{j}\right) \Psi
$$

where

$$
\begin{aligned}
& \mid \Psi>=\Omega^{-(N+1) / 2} \exp (i \boldsymbol{q} \cdot \boldsymbol{r}) \exp \left(i \boldsymbol{Q}_{1} \cdot \boldsymbol{R}_{1}\right) \ldots \exp \left(i \boldsymbol{Q}_{N} \cdot \boldsymbol{R}_{N}\right) \\
& \mid \Psi^{\prime}>=\Omega^{-(N+1) / 2} \exp [i(\boldsymbol{q}+\boldsymbol{K}) \cdot \boldsymbol{r}] \exp \left(i \boldsymbol{Q}_{1}{ }^{\prime} \cdot \boldsymbol{R}_{1}\right) \ldots \exp \left(i \boldsymbol{Q}_{N}{ }^{\prime} \cdot \boldsymbol{R}_{N}\right)
\end{aligned}
$$

and $\boldsymbol{Q}_{1}{ }^{\prime}, \ldots \boldsymbol{Q}_{N}{ }^{\prime}$ indicate the wavevectors of the solvent atoms after the above interaction. Therefore 


$$
\begin{aligned}
& <\Psi^{\prime}\left|H^{\prime}\right| \Psi>= \\
& \Omega^{-(N+1)} \int \mathrm{d}^{3} r \int \mathrm{d}^{3} R_{1} \ldots \int \mathrm{d}^{3} R_{N} \exp \left[i\left(\boldsymbol{Q}_{1}-\boldsymbol{Q}_{1}{ }^{\prime}\right) \cdot \boldsymbol{R}_{1}+\ldots i\left(\boldsymbol{Q}_{N}-\boldsymbol{Q}_{N}{ }^{\prime}\right) \cdot \boldsymbol{R}_{N}\right] \\
& \quad \exp (-i \boldsymbol{K} \cdot \boldsymbol{r})\left[\varphi\left(\boldsymbol{r}-\boldsymbol{R}_{1}\right)+\ldots \varphi\left(\boldsymbol{r}-\boldsymbol{R}_{N}\right)\right]
\end{aligned}
$$

For each term $\varphi\left(\boldsymbol{r}-\boldsymbol{R}_{j}\right)$ appearing in the second square bracket one can write

$$
\int \mathrm{d}^{3} r \exp (-i \boldsymbol{K} \cdot \boldsymbol{r}) \varphi\left(\boldsymbol{r}-\boldsymbol{R}_{j}\right)=\Phi(K) \exp \left(-i \boldsymbol{K} \cdot \boldsymbol{R}_{j}\right)
$$

The cyclic boundary conditions imposed to the box of volume $\Omega$ give

$$
\int \mathrm{d}^{3} R_{j} \exp \left[i\left(\boldsymbol{Q}-\boldsymbol{Q}^{\prime}\right) \cdot \boldsymbol{R}_{j}\right]=\Omega \delta\left(\boldsymbol{Q}, \boldsymbol{Q}^{\prime}\right)
$$

where $\delta\left(\boldsymbol{Q}, \boldsymbol{Q}^{\prime}\right)$ is the Kroenecker symbol. Consequently the probability rate corresponding to the matrix element (A1) is

$$
\begin{gathered}
\left(2 \pi / \hbar \Omega^{2}\right) \Phi^{2}(K) \Sigma_{1}{ }^{\prime} \Sigma_{2}{ }^{\prime} \ldots \Sigma_{N}{ }^{\prime}[ \\
\delta\left(\boldsymbol{Q}_{1}{ }^{\prime}, \boldsymbol{Q}_{1}-\boldsymbol{K}\right) \delta\left(\boldsymbol{Q}_{2}{ }^{\prime}, \boldsymbol{Q}_{2}\right) \ldots \delta\left(\boldsymbol{Q}_{N}{ }^{\prime}, \boldsymbol{Q}_{N}\right)+ \\
\delta\left(\boldsymbol{Q}_{1}{ }^{\prime}, \boldsymbol{Q}_{1}\right) \delta\left(\boldsymbol{Q}_{2}{ }^{\prime}, \boldsymbol{Q}_{2}-\boldsymbol{K}\right) \ldots \delta\left(\boldsymbol{Q}_{N}{ }^{\prime}, \boldsymbol{Q}_{N}\right)+ \\
\left.\ldots\left(\boldsymbol{Q}_{1}{ }^{\prime}, \boldsymbol{Q}_{1}\right) \delta\left(\boldsymbol{Q}_{2}{ }^{\prime}, \boldsymbol{Q}_{2}\right) \ldots \delta\left(\boldsymbol{Q}_{N}{ }^{\prime}, \boldsymbol{Q}_{N}-\boldsymbol{K}\right)\right]^{2} \\
\delta\left[\varepsilon_{a}(\boldsymbol{q})+\varepsilon_{b}\left(\boldsymbol{Q}_{1}\right)+\varepsilon_{b}\left(\boldsymbol{Q}_{2}\right)+\ldots \varepsilon_{b}\left(\boldsymbol{Q}_{N}\right)-\varepsilon_{a}(\boldsymbol{q}+\boldsymbol{K})-\varepsilon_{b}\left(\boldsymbol{Q}_{1}{ }^{\prime}\right)-\varepsilon_{b}\left(\boldsymbol{Q}_{2}{ }^{\prime}\right)-\ldots \varepsilon_{b}\left(\boldsymbol{Q}_{N}{ }^{\prime}\right)\right]
\end{gathered}
$$

where $\varepsilon_{s}(\boldsymbol{q})=\hbar^{2}|\boldsymbol{q}|^{2} / 2 m_{s}$ and the sum is over all the allowed final states of the host liquid (we have used the notation $\Sigma_{j}{ }^{\prime}$ to indicate the sum over all the vectors $\boldsymbol{Q}_{j}{ }^{\prime}$ of the $j^{\text {th }}$ atom). For any $\boldsymbol{K} \neq 0$ all the interference terms required by the square of the bracket vanish: for instance, the interference between the first two terms involves the product $\delta\left(\boldsymbol{Q}_{1}{ }^{\prime}, \boldsymbol{Q}_{1}-\boldsymbol{K}\right) \delta\left(\boldsymbol{Q}_{1}{ }^{\prime}, \boldsymbol{Q}_{1}\right)$ which is clearly zero. The result is precisely eq. (1) of the text.

\section{Appendix B.}

Starting from eq. (9), let us expand $\exp (-\mathcal{K})$ in powers of $u$ and divide both sides of the equation by $u$ : in this way we obtain

$$
\int_{0}^{\infty} y B(y) \exp \left(-\alpha y^{2}\right) \mathrm{d} y \int_{-1}^{1}[1-(1+\mu) y u \xi+\ldots][G(u)-(1+y \xi / u) G(t)] \mathrm{d} \xi=1
$$

where $\alpha=(1+\mu)^{2} / 4 \mu$. Performing the limit for $u \rightarrow 0$ and noting that 


$$
\begin{aligned}
\lim \int_{-1}^{1} \mathrm{~d} \xi \xi u^{-1} G(t) & =\lim \int_{-1}^{1} \mathrm{~d} \xi \xi u^{-1}[G(t)-G(y)]=\lim \int_{-1}^{1} \mathrm{~d} \xi \xi G^{\prime}(t)(\partial t / \partial u) \\
& =\int_{-1}^{1} \mathrm{~d} \xi \xi^{2} G^{\prime}(y)=(2 / 3) G^{\prime}(y)
\end{aligned}
$$

we arrive at the relation

$$
\begin{aligned}
& 2 G(0) \int_{0}^{\infty} \mathrm{d} y y B(y) \exp \left(-\alpha y^{2}\right)= \\
& 1+2 \int_{0}^{\infty} \mathrm{d} y y B(y)\left\{G(y)+\left[y G^{\prime}(y)-(1+\mu) y^{2} G(y)\right] / 3\right\} \exp \left(-\alpha y^{2}\right)
\end{aligned}
$$

$G(0)$ depends in principle on $\lambda_{a}$ and $\mu$ : since no singularity is present in the integrand on the right hand side of eq. (B3), it is reasonable to consider $G(0)$ as an anlytical function of these variables. It is easy to show that this function cannot be identically zero. In fact for $G(0)=0$ we obtain from eq. (B3), after an integration by parts,

$$
\int_{0}^{\infty} \mathrm{d} y \text { y } B(y) G(y)\left[1-y L(y)+y^{2}\left(1-\mu^{2}\right) / 2 \mu\right] \exp \left(-\alpha y^{2}\right)=-3 / 2
$$

where the function $L(y)=B^{\prime}(y) / B(y)$ is negative, owing to the fact that in the low $K$ region $\Phi(K)$ is a strongly decreasing function of its argument. In the range $\mu \leq 1$ (which includes the important case of self-diffusion) the square bracket of eq. (B4) is positive, so that this equation cannot be satisfied unless $G(y)$ is negative in some interval of the $y$ axis. Inconsistencies of this kind can only be avoided by assuming the existence of positive values of $G(0)$ in the above range. Because of the assumption of analyticity, the curve representing $G(0)$ vs $\mu$ for $\mu \leq 1$ must continue in the plane $\mu>1$. Such a curve associates to any value of $\mu$ a corresponding numerical value of $G(0)$ : when this is inserted into eq. (8) $\psi_{q}$ takes the form of the trial function used in $\mathrm{I}$, which is therefore justified in the long wavelength limit.

\section{Appendix C.}

With reference to the integral equation (9), let us examine the high $\boldsymbol{q}$ behaviour of the solution $(u \rightarrow \infty)$. To this purpose, let us discuss the conditions under which a function of the form $u^{v}$ can represent the asymptotic behaviour of $G(u)$. Using the expansion

$$
\exp (-\mathcal{K})=\left[\exp \left(-\alpha y^{2}-\mu u^{2} \xi^{2}\right)\right] \sum_{n=0}^{\infty}(-1)^{n}(n !)^{-1}(1+\mu)^{n}\left(\begin{array}{lll}
u & y & \xi
\end{array}\right)^{n}
$$


where $\alpha$ is defined as in Appendix B, we easily obtain, for $G(u)=u^{v}$,

$u G(u) \int_{-1}^{1} \exp (-\mathcal{K}) \mathrm{d} \xi=\left[\exp \left(-\alpha y^{2}\right)\right] u^{v} \sum_{n=0}^{\infty}(1+\mu)^{2 n}\left[y^{2 n} /(2 n) !\right] \mu^{-(n+1 / 2)} \Gamma(n+1 / 2)$

having noted that for $u \rightarrow \infty$ the integral over $\xi$ can be extended from $-\infty$ to $+\infty$. In a similar way, considering that in the above hypothesis

$$
\begin{aligned}
& G(t)=[1+(y / u)(2 \xi+y / u)]^{v / 2} u^{v}= \\
& u^{v} \sum_{k=0}^{\infty}(v / 2, k) \sum_{s=0}^{k}(k, s)(2 \xi)^{k-s} y^{k+s} u^{-(k+s)}
\end{aligned}
$$

where $(s, k)=s ! /[k !(s-k) !]$, we find

$$
\begin{aligned}
& \int_{-1}^{1}(u+y \xi) G(t) \exp (-\mathcal{K}) \mathrm{d} \xi=\left[\exp \left(-\alpha y^{2}\right)\right] \sum_{k=0}^{\infty} u^{v-2 k} \sum_{n=0}^{\infty}(-1)^{n}(n !)^{-1}(1+\mu)^{n} \\
& \sum_{s=0}^{k}(v / 2, k)(k, s) 2^{k-s} \Gamma[(n+k-s+1) / 2] \mu^{-(n+k-s+1) / 2} y^{n+k+s}+ \\
& (1 / 2)\left[\exp \left(-\alpha y^{2}\right)\right] \sum_{k=1}^{\infty} u^{v-2 k} \sum_{n=0}^{\infty}(-1)^{n}(n !)^{-1}(1+\mu)^{n} \sum_{s=0}^{k-1}(v / 2, k-1)(k-1, s) 2^{k-s} \\
& \Gamma[(n+k-s+1) / 2] \mu^{-(n+k-s+1) / 2} y^{n+k+s}
\end{aligned}
$$

where $n$ is subject to the condition $n+k-s=$ even integer.

The two expressions (C2) and (C4) must be subtracted, as required by the right hand side of the integral equation (9), so that the contribution of $k=0$ to the first term of eq. (C4) cancels exactly the integral (C2). The final result is therefore obtainable from eq. (C4) by putting $k=1$ as a lower limit in both the terms appearing on its right hand side, which in this way becomes expressible as a linear combination of the descending powers $u^{v-2}, u^{v-4}, \ldots$. Such an expansion for $u \rightarrow \infty$, when multiplied by $y B(y)$ and integrated over $y$, can represent the left hand side of the integral equation (9) only if $v=3$. In other words, the asymptotic form of $G(u)$ must be proportional to $u^{3}$.

\section{Appendix D.}

Let us consider a particle of mass $m_{a}$ with wavevector $\boldsymbol{q}$, interacting with a solid composed of $N$ atoms of mass $m_{b}$. Let $\Phi(K)$ be the Fourier transform of the pair potential describing the interaction between the particle and one of the atoms of the solid. The matrix element for a scattering $\boldsymbol{q} \rightarrow \boldsymbol{q}^{\prime}$ in which the particle undergoes a wavevector change $\boldsymbol{K}=\boldsymbol{q}^{\prime}-\boldsymbol{q}$ is

$$
\Omega^{-1} \Phi(K) \Sigma_{j} \exp \left[-i \boldsymbol{K} \cdot\left(\boldsymbol{R}_{j}+\boldsymbol{\eta}_{j}\right)\right] \cong \Omega^{-1} \Phi(K) \Sigma_{j}\left(1-i \boldsymbol{K} \cdot \boldsymbol{\eta}_{j}\right) \exp \left(-i \boldsymbol{K} \cdot \boldsymbol{R}_{j}\right)
$$


where $\boldsymbol{\eta}_{j}$ is the displacement of the $j^{\text {th }}$ atom from its equilibrium position $\boldsymbol{R}_{j}$. For any $\boldsymbol{K}$ different from a reciprocal lattice vector the term with unity in the square bracket vanishes, owing to the periodic boundary conditions imposed to the lattice. Let us introduce the probability rate $\mathcal{P}\left(\boldsymbol{q}, \boldsymbol{Q}_{p} ; \boldsymbol{q}^{\prime}\right)$ for a scattering process in which the particle with wavevector $\boldsymbol{q}$, interacting with a phonon of wavevector $\boldsymbol{Q}$ and polarization index $p$, acquires a final wavevector $\boldsymbol{q}^{\prime}:$ the probability rate for the inverse process is denoted by $\mathcal{P}\left(\boldsymbol{q}^{\prime} ; \boldsymbol{q}, \boldsymbol{Q}_{p}\right)$ and, for the principle of microscopic reversibility, is equal to $\mathcal{P}\left(\boldsymbol{q}, \boldsymbol{Q}_{p} ; \boldsymbol{q}^{\prime}\right)$. For $\boldsymbol{K}$ sufficiently small, using the expansion of $\eta_{j}$ in terms of creation and absorption phonon operators, it is easy to arrive at the following expression

$$
P\left(\boldsymbol{q}, \boldsymbol{Q}_{p} ; \boldsymbol{q}^{\prime}\right)=2 \pi \Omega^{-2} \Phi^{2}(K)\left(N / 2 m_{b} \omega_{\boldsymbol{Q} p}\right)\left(\boldsymbol{K} \cdot \boldsymbol{e}_{\boldsymbol{Q} p}\right)^{2} \delta(\boldsymbol{K}, \boldsymbol{Q}) \delta\left(\varepsilon_{q^{\prime}}-\varepsilon_{q}\right)
$$

where $\boldsymbol{e}_{\boldsymbol{Q} p}$ is the polarization unit vector of the phonon, $\omega_{\boldsymbol{Q}_{p}}$ its angular frequency and $\varepsilon_{q}=\hbar^{2} q^{2} / 2 m_{a}$. Since $\boldsymbol{K}$ was assumed to be small, a phonon energy term $\hbar \omega_{\boldsymbol{K} p}$ has been neglected in the argument of the delta function. In an isotropic solid, the condition $\boldsymbol{K}=\boldsymbol{Q}$ implies that the factor $\left(\boldsymbol{K} \cdot \boldsymbol{e}_{\boldsymbol{Q}_{p}}\right)^{2}$ survives only if $p$ is the index of a longitudinal phonon. This suggests to denote hereafter a probability rate by the symbol $\mathcal{P}\left(\boldsymbol{q}, \boldsymbol{Q} ; \boldsymbol{q}^{\prime}\right)$, where $\boldsymbol{Q}$ is understood to refer to a longitudinal phonon.

Let us now consider a system of identical particles with mass $m_{a}$, undergoing a diffusion process through the solid, and let $f_{q}$ be the number of these particles with wavevector $\boldsymbol{q}$ per unit volume. The contribution to the time derivative of $f_{\boldsymbol{q}}$, as due to the interaction of these particles with the phonon field, is easily seen to be

$$
\begin{aligned}
\partial f_{\boldsymbol{q}} / \partial t= & \Sigma_{\boldsymbol{q}^{\prime}, \boldsymbol{Q}} \mathcal{P}\left(\boldsymbol{q}^{\prime}, \boldsymbol{Q} ; \boldsymbol{q}\right) n_{\boldsymbol{Q}} f_{q^{\prime}}-\Sigma_{\boldsymbol{q}^{\prime}, \boldsymbol{Q}} \mathcal{P}\left(\boldsymbol{q} ; \boldsymbol{q}^{\prime}, \boldsymbol{Q}\right) f_{\boldsymbol{q}}\left(1+n_{\boldsymbol{Q}}\right)+ \\
& \Sigma_{\boldsymbol{q}^{\prime}, \boldsymbol{Q}} \mathcal{P}\left(\boldsymbol{q}^{\prime} ; \boldsymbol{q}, \boldsymbol{Q}\right) f_{\boldsymbol{q}^{\prime}}\left(1+n_{\boldsymbol{Q}}\right)-\Sigma_{\boldsymbol{q}^{\prime}, \boldsymbol{Q}} \mathcal{P}\left(\boldsymbol{q}, \boldsymbol{Q} ; \boldsymbol{q}^{\prime}\right) f_{\boldsymbol{q}} n_{\boldsymbol{Q}}
\end{aligned}
$$

where $n_{Q}$ is the number of longitudinal phonons with wavevector $\boldsymbol{Q}$. In the high temperature limit $n_{Q}>>1$, so that

$$
\partial f_{\boldsymbol{q}} / \partial t=\Sigma_{\boldsymbol{q}^{\prime}, \boldsymbol{Q}}\left[P\left(\boldsymbol{q}^{\prime}, \boldsymbol{Q} ; \boldsymbol{q}\right)+P\left(\boldsymbol{q}^{\prime} ; \boldsymbol{q}, \boldsymbol{Q}\right)\right]\left(f_{\boldsymbol{q}^{\prime}}-f_{\boldsymbol{q}}\right) n_{\boldsymbol{Q}}
$$

The two terms in the square bracket are seen to produce equal contributions, so that

$$
\partial f_{q} / \partial t=\left(2 \pi / m_{b} N v^{2}\right) \Sigma_{q^{\prime}} \omega_{K}^{-1} K^{2} \Phi^{2}(K) n_{K}\left(f_{q^{\prime}}-f_{q}\right) \delta\left(\varepsilon_{q^{\prime}}-\varepsilon_{q}\right)
$$

At high temperatures $n_{K} \cong k_{\mathrm{B}} T / \hbar \omega_{K}=k_{\mathrm{B}} T / \hbar s_{K} K$, where $s_{K}$ is the longitudinal sound velocity. Transforming the sum over $\boldsymbol{q}^{\prime}$ into an integral and using the properties of the delta function, one easily obtains

$$
\partial f_{q} / \partial t=\left(k_{\mathrm{B}} T m_{a} / 4 \pi^{2} v \hbar^{3} m_{b}\right) q \int \mathrm{d} \Omega^{\prime} s_{K}^{-2} \Phi^{2}(K)\left(f_{q^{\prime}}-f_{q}\right)
$$

where $\mathrm{d} \Omega^{\prime}=\sin \theta^{\prime} \mathrm{d} \theta^{\prime} \mathrm{d} \phi^{\prime}$ is the elementary solid angle defining the orientation of $\boldsymbol{q}^{\prime}$ in a reference frame with polar axis $\boldsymbol{q}$ and $K=2 q \sin \left(\theta^{\prime} / 2\right)$. 
The assumption that phonon modes are present in liquids amounts in particular to extending to these systems the validity of eq. (D6). This has two important consequences.

a) To discuss the first consequence, let us think of an alternative description, in which the liquid is considered as a system of particles without collective motions. Since $\Phi(K)$ is confined to a short interval near $K=0$, the scattering processes of the diffusing particles (due to their interaction with the solvent atoms) are approximately elastic, so that it is possible to define a probability rate $Q\left(\boldsymbol{q}, \boldsymbol{q}^{\prime}\right)$ with $\left|\boldsymbol{q}^{\prime}\right|=|\boldsymbol{q}|$. The corresponding expression of $\partial f_{q} / \partial t$ is

$$
\partial f_{\boldsymbol{q}} / \partial t=\Sigma_{\boldsymbol{q}^{\prime}} Q\left(\boldsymbol{q}, \boldsymbol{q}^{\prime}\right)\left(f_{\boldsymbol{q}^{\prime}}-f_{\boldsymbol{q}}\right)
$$

where

$$
Q\left(\boldsymbol{q}, \boldsymbol{q}^{\prime}\right)=\left(2 \pi / \hbar \Omega^{2}\right) \Phi^{2}(K)\left|\Sigma_{j} \exp \left(-i \boldsymbol{K} \cdot \boldsymbol{R}_{j}\right)\right|^{2} \delta\left(\varepsilon_{\boldsymbol{q}^{\prime}}-\varepsilon_{\boldsymbol{q}}\right)
$$

and the sum over $j$ is extended to all the position vectors $\boldsymbol{R}_{j}$ of the solvent atoms. The square modulus appearing in eq. (D8) is $N a_{K}$, so that, transforming the sum over $\boldsymbol{q}^{\prime}$ into an integral, one has

$$
\partial f_{q} / \partial t=\left(m_{a} q / 4 \pi^{2} v \hbar^{3}\right) \int \mathrm{d} \Omega^{\prime} a_{K} \Phi^{2}(K)\left(f_{q^{\prime}}-f_{q}\right)
$$

The two equations (D6) and (D9) are identical provided one assumes

$$
s_{K}^{2}=k_{\mathrm{B}} T /\left(m_{b} a_{K}\right)
$$

Such a result throws light on the function $\Phi(K)$ describing the interaction between the diffusing particle and an atom of the host liquid. When $\Phi(K)$ is a short range function, confined to a small interval near $K=0$, one can always interpret the scattering processes undergone by the diffusing particle as due to the interaction between this particle and a system of phonons characterized by wavevectors lying in the above interval and velocity satisfying eq. (D10). This equation is coincident with the well known dispersion relation for the isothermal modes predictable in the frame of Percus-Yevick theory (Ascarelli et al., [12]).

$b)$ The second consequence is the determination of the mean free path due to phonon scattering. Since $\left|\boldsymbol{q}^{\prime}\right|=|\boldsymbol{q}|$, eq. (D6) is clearly consistent with the existence of a relaxation time $\tau_{q}$ defined by the equation

$$
1 / \tau_{q}=\left(k_{\mathrm{B}} T m_{a} q / 4 \pi^{2} v \hbar^{3} m_{b} s_{0}^{2}\right) \int \mathrm{d} \Omega^{\prime}\left(1-\cos \theta^{\prime}\right) \Phi^{2}\left[2 q \sin \left(\theta^{\prime} / 2\right)\right]
$$

where $s_{0}$ is the sound velocity corresponding to eq. (D10) for $K=0$ and $\Phi^{2}(K)$ is represented by the first term of eq. (25), namely $\Phi^{2}(0) Y\left(x^{\prime}\right)$, with $x^{\prime}=\left(2 q / K_{m}\right)$ $\sin \left(\theta^{\prime} / 2\right)$. For $m_{a}=m_{b}=m$, using $x^{\prime}$ as the integration variable, one easily obtains for the mean free path $l_{q}=V_{q} \tau_{q}$ the equation 


$$
1 / l_{q}=\left(k_{\mathrm{B}} T m / 4 \pi v \hbar^{4} s_{0}{ }^{2}\right) \Phi^{2}(0)\left(K_{m} / q\right)^{4} J \cong\left[v\left(m k_{\mathrm{B}} T\right)^{2} / 4 \pi \hbar^{4} a_{0}\right]\left(K_{m} / q\right)^{4} J
$$

where eqs. (18) and (D10) have been used, while the presence of $J$ (see eq. (30)) follows from the assumption $q>K_{m} / 2$, largely satisfied for the value of $q$ corresponding to the average thermal energy, $q_{t h}=\left(3 k_{\mathrm{B}} T m\right)^{1 / 2} / \hbar$. In this case the mean free path is given by the expression

$$
l_{\text {th }}=36 \pi a_{0} /\left(v K_{m}^{4} J\right)
$$

and amounts to $7.05 \AA$ for $\mathrm{Li}^{7}$ at the melting point. As expected, this is much higher than the local interaction contribution $(0.154 \AA)$.

Acknowledgement: This work would not have been accomplished without the constant encouragement of my wife, to whom all my gratitude is addressed.

\section{REFERENCES}

[1] M. Omini, Phil. Mag., A54, 561, 1986

[2] M. Omini, Phil. Mag., A57, 799, 1988

[3] A. Feinauer, G. Majer and A. Seeger, J. Phys: Condens. Matter, 6, L355, 1994

[4] A. Feinauer, G. Majer and A. Seeger, Defect and Diffusion Forum, 143-147, 881, 1997

[5] J. Buchhauser, T. Gross, N. Karger and H.D. Lüdermann, J. Chem. Phys., 110, 3037, 1999

[6] A.I. Gubanov, Quantum Electron Theory of Amorphous Conductors, Consultants Bureau, New York, 1965

[7] M. Goda and S. Osabe, J. Phys. F: Met. Phys., 8, 1019, 1978

[8] F.S. Gaeta, C. Albanese, D.G. Mita and F. Peluso, Phys. Rev. E 49, 433, 1994

[9] J.M. Ziman, Phil. Mag., 6, 1013, 1961

[10] P.A. Egelstaff, Adv. Phys., 16, 147, 1967

[11] H.Sinn and E. Burkel, J. Phys.: Condens. Matter, 8, 9369, 1996

[12] P. Ascarelli, R.J. Harrison and A. Paskin, Adv. Phys., 16, 717, 1967

[13] T.E. Faber, Introduction to the Theory of Liquid Metals, Cambridge University Press, 1972

[14] M. Shimoji, Liquid Metals, Academic Press, New York, 1977

[15] T. Iida and R.I.L. Guthrie, The Physical Properties of Liquid Metals, Clarendon Press, Oxford, 1988, p. 71. 


\begin{tabular}{|c|c|c|c|c|c|c|c|c|c|}
\hline & $T_{m}$ & $v$ & $\sigma$ & $a_{0}$ & $\eta$ & $K_{m} / Q_{\mathrm{D}}$ & $\left(D^{s / s}\right)_{\exp }$ & $l$ & $l / R_{A}$ \\
\hline \multirow[t]{2}{*}{$\mathrm{Li}^{7}$} & 454 & 22.72 & 2.648 & 0.031 & 0.428 & 0.39 & 6.1 & 1.82 & 0.10 \\
\hline & & & & & & & 5.64 & 1.54 & 0.09 \\
\hline $\mathrm{Na}$ & 373 & 41.19 & 3.298 & 0.024 & 0.456 & 0.36 & 4.2 & 4.14 & 0.19 \\
\hline $\mathrm{K}$ & 338 & 78.90 & 4.108 & 0.023 & 0.460 & 0.36 & 3.7 & 4.92 & 0.19 \\
\hline
\end{tabular}

Table 1. Parameters of interest for lithium, sodium and potassium at the melting point (see text): $v$ is given in $\AA^{3}, \sigma$ in $\AA, D^{s / s}$ in $10^{-9} \mathrm{~m}^{2} \mathrm{~s}^{-1}, l$ in $10^{-1} \AA$.

$\begin{array}{llll} & \mu=1 & \mu=7 / 6 & \mu=6 / 7 \\ ---------- & \\ R_{1} & 0.627 & 0.602 & 0.650 \\ R_{2} & 1.679 & 1.538 & 1.824 \\ R_{3} & 15.63 & 13.57 & 17.92 \\ S_{1} & 1.253 & 1.397 & 1.108 \\ S_{2} & 6.511 & 7.326 & 5.700 \\ S_{3} & 71.48 & 78.66 & 64.15\end{array}$

Table 2 . Numerical values of the functions defined by eqs. (33)-(35) and (37)-(39). 